OPEN ACCESS

Edited by:

Benjamin Andrew Evans,

University of East Anglia,

United Kingdom

Reviewed by:

Rolf Kümmerli,

University of Zurich, Switzerland

Lorenzo Segovia,

Universidad Nacional Autónoma

de México, Mexico

*Correspondence:

Manja Saebelfeld msaebelfeld@gmail.com

Suman G. Das

sdas3@uni-koeln.de

Specialty section:

This article was submitted to Antimicrobials, Resistance

and Chemotherapy,

a section of the journal

Frontiers in Microbiology

Received: 22 April 2021

Accepted: 29 July 2021

Published: 19 August 2021

Citation:

Saebelfeld M, Das SG, Brink J,

Hagenbeek A, Krug J and

de Visser JAGM (2021) Antibiotic

Breakdown by Susceptible Bacteria

Enhances the Establishment

of $\beta$-Lactam Resistant Mutants.

Front. Microbiol. 12:698970.

doi: 10.3389/fmicb.2021.698970

\section{Antibiotic Breakdown by Susceptible Bacteria Enhances the Establishment of $\beta$-Lactam Resistant Mutants}

\author{
Manja Saebelfeld ${ }^{1,2 *}$, Suman G. Das ${ }^{1 *}$, Jorn Brink ${ }^{2}$, Arno Hagenbeek ${ }^{2}$, Joachim Krug \\ and J. Arjan G. M. de Visser ${ }^{2}$
}

1 Institute for Biological Physics, University of Cologne, Cologne, Germany, ${ }^{2}$ Laboratory of Genetics, Department of the Plant Sciences Group, Wageningen University and Research, Wageningen, Netherlands

For a better understanding of the evolution of antibiotic resistance, it is imperative to study the factors that determine the initial establishment of mutant resistance alleles. In addition to the antibiotic concentration, the establishment of resistance alleles may be affected by interactions with the surrounding susceptible cells from which they derive, for instance via the release of nutrients or removal of the antibiotic. Here, we investigate the effects of social interactions with surrounding susceptible cells on the establishment of Escherichia coli mutants with increasing $\beta$-lactamase activity (i.e., the capacity to hydrolyze $\beta$-lactam antibiotics) from single cells under the exposure of the antibiotic cefotaxime (CTX) on agar plates. We find that relatively susceptible cells, expressing a $\beta$-lactamase with very low antibiotic-hydrolyzing activity, increase the probability of mutant cells to survive and outgrow into colonies due to the active breakdown of the antibiotic. However, the rate of breakdown by the susceptible strain is much higher than expected based on its low enzymatic activity. A detailed theoretical model suggests that this observation may be explained by cell filamentation causing delayed lysis. While susceptible cells may hamper the spread of higher-resistant $\beta$-lactamase mutants at relatively high frequencies, our findings show that they promote their initial establishment.

\section{Keywords: Escherichia coli, $\beta$-lactamase, social interactions, mutant establishment, resistance evolution}

\section{INTRODUCTION}

Antibiotic resistance has become a worldwide concern, causing 700,000 deaths annually due to failed treatments (IACG, 2019). As the development of novel drugs does not appear to be promising in the long-term, other strategies of slowing or halting the emergence of antibiotic resistance have been explored, including diverse prevention strategies (e.g., rational use of antibiotics and infection control), the use of biologics or adjuvants to disturb quorum sensing of resistant bacteria, the use of bacteriophages (reviewed in Aslam et al., 2018) and the exploitation of the evolutionary potential and interactions of resistance to develop new treatment strategies (Schenk et al., 2012; Palmer and Kishony, 2013; Baym et al., 2016; Furusawa et al., 2018). 
For a better understanding of the evolution of antibiotic resistance, it is not only important to study the spread of resistant bacteria, but also the factors that determine the emergence of new resistance alleles in the first place (Alexander and MacLean, 2020). Resistance alleles can be acquired via mutation or horizontal gene transfer (Palmer and Kishony, 2013; Blair et al., 2015). In either case, the resistant allele is initially present at a very low frequency and thus prone to random extinction. Population genetics theory predicts that only when an allele has survived extinction by genetic drift and reaches an absolute frequency roughly equal to the inverse of its selection coefficient, its course becomes dominated by selection in an environment where it is beneficial (Haldane, 1927; Patwa and Wahl, 2008). Mutations that have survived genetic drift are often called "established" (Levy et al., 2015; Alexander and MacLean, 2020), although they may still be driven to extinction by the competition with other mutants (Gerrish and Lenski, 1998; Rozen et al., 2002). The few existing empirical studies on the establishment of rare alleles have shown that this stochastic process of "drift loss" is influenced by the initial allele frequency and the fitness benefit of the allele (Chelo et al., 2013; Gifford et al., 2013).

Only a handful of studies so far have systematically investigated the establishment of single bacterial cells expressing an antibiotic-resistant allele. Those studies found that the probability of a resistant mutant to establish depends on the type and concentration of nutrients (Coates et al., 2018) and antibiotics (Coates et al., 2018; Alexander and MacLean, 2020). Alexander and MacLean (2020) found that the establishment probability (i.e., outgrowth into a visible population) of a single streptomycin-resistant Pseudomonas aeruginosa cell was only $5 \%$ at a concentration as low as $1 / 8$ of the strains' minimum inhibitory concentration (MIC) in the absence of a wildtype population. Interestingly, when the same mutant was introduced into a large population of wildtype cells, its establishment was strongly increased under the same streptomycin concentrations. The authors speculated that this effect was due to the removal of the antibiotic from the environment via binding to wildtype cell components (Alexander and MacLean, 2020).

Determining factors that influence the establishment of antibiotic-resistance alleles could have implications in a clinical context, for instance by adjusting drug dosing strategies to a point where the establishment of de novo mutations is strongly reduced (Alexander and MacLean, 2020). For this, interactions between newly arising resistance alleles and nearby sensitive cells must be understood better. Apart from drug removal by binding to cellular components, other common goods, such as enzymes causing antibiotic hydrolysis or the release of nutrients from killed cells, may play a role. A good model system to study such social interactions is TEM-1 $\beta$-lactamase. This enzyme is expressed in the periplasmic space of the bacterial cell wall, where it hydrolyses $\beta$-lactam antibiotics such as penicillins. This leads to a reduction of the antibiotic concentration in the environment and may support the growth of more susceptible cells (Brown et al., 2009; Medaney et al., 2016; Nicoloff and Andersson, 2016). If and to what extent such interactions affect the establishment of $\beta$-lactamase mutants conferring higher resistance, is unknown.
Here, we tested the effect of relatively antibiotic-susceptible cells, expressing the ancestral TEM- $1 \beta$-lactamase allele with a very low antibiotic-hydrolyzing capacity, on the establishment of higher-resistance TEM alleles. In particular, we distinguished between possible effects of increased nutrient availability due to cell lysis and drug removal via $\beta$-lactamase activity or binding to antibiotic target sites. We used the $\beta$-lactam antibiotic cefotaxime (CTX) and different strains of the bacterium Escherichia coli, expressing $\beta$-lactamase alleles with varying activity against CTX. We simulated the establishment process of CTX-resistant E. coli by introducing a low number of mutant cells into populations of more susceptible, yet antibiotic-hydrolyzing, cells on CTXcontaining agar plates. Our results revealed substantial increases in mutant establishment probability in the presence of relatively susceptible cells. By using heat-killed cells and measuring the CTX-reducing capacity of $\beta$-lactamase-expressing strains compared to the wildtype without $\beta$-lactamase, we distinguished between the effects from nutrient addition by cell lysis and antibiotic removal via either CTX binding or hydrolysis on mutant establishment. Lastly, to understand an unexpectedly large effect on mutant establishment by the weakly $\beta$-lactamaseexpressing ancestral strain, we used a detailed dynamical model. This model suggests that certain population dynamic parameters, such as cell filamentation and $\beta$-lactamase synthesis rate, may have played a crucial role during the establishment of the higherresistance mutants.

\section{MATERIALS AND METHODS}

\section{Strains and Culture Conditions}

Several strains, derived from E. coli strain MG1655 were used for the experiments. This strain had previously been modified with either a yellow fluorescent protein (YFP) or a blue fluorescent protein (BFP) chromosomal marker cassette, containing a resistance gene for chloramphenicol (Gullberg et al., 2014). For the current study, the chloramphenicol resistance was removed to serve as the non- $\beta$-lactamase-expressing Wildtype strain (Table 1). Into each of the two fluorescently marked Wildtype strains, one of four TEM variants was inserted into the galK locus: the ancestral TEM-1 allele (from now on referred to as Ancestor), and three mutant alleles with either 1, 2, or 3 point mutations in the TEM gene (from now on referred to as "Single mutant," "Double mutant" and, "Triple mutant," respectively; cf. Table 1). While the ancestral TEM-1 allele confers very low activity toward CTX, the three mutants show increasing CTX resistance with each additional substitution (Table 1). All TEM loci are under the control of the lacI repressor and are expressed by adding $50 \mu \mathrm{M}$ Isopropyl $\beta$-D-1-thiogalactopyranoside (IPTG) to the growth medium. The MIC for CTX was determined per strain in duplicates (for each of the YFP and BFP variants), using 2-fold increases in CTX concentration in microtiter plates filled with $200 \mu \mathrm{l}$ M9 minimal medium (containing 0.4\% glucose, $0.2 \%$ casamino acids, $2 \mu \mathrm{g} / \mathrm{ml}$ uracil, and $1 \mu \mathrm{g} / \mathrm{ml}$ thiamine) and $50 \mu \mathrm{M}$ IPTG, inoculated with $10^{5}$ cells and incubated for $24 \mathrm{~h}$ at $37^{\circ} \mathrm{C}$ (cf. Table $\mathbf{1}$ ). 
For setting up the experiments, all strains were grown overnight at $37^{\circ} \mathrm{C}$ and $250 \mathrm{rpm}$ in $1-2 \mathrm{ml} \mathrm{M} 9$ medium. The cultures were either directly inoculated from the $-80^{\circ} \mathrm{C}$ glycerol stocks or first streaked out on Luria-Bertani (LB) agar plates from which a single colony was picked into the liquid M9 medium. After overnight incubation, the cultures were serially diluted with phosphate-buffered saline (PBS) to the density needed for the particular experiment (see below). For the Interaction experiments on agar, the diluted cultures (or culture mixtures) were spread on $92 \mathrm{~mm}$ plates containing M9 medium (as above) with $1.5 \%$ agar, $50 \mu \mathrm{M}$ IPTG, and the respective CTX concentration as specified by the particular experiment (see below).

\section{Interaction Experiment 1}

First, we tested whether the previous observation that the establishment, i.e., the stochastic process of a single cell surviving drift and outgrowing into a population, of an antibiotic-resistant mutant increases in the presence of susceptible cells (Alexander and MacLean, 2020), also applies to our TEM $\beta$-lactamase system. For this, roughly 150 cells each of the three E. coli TEM mutants (Table 1) were introduced into populations of relatively susceptible bacteria expressing the ancestral TEM1 allele with very low $\beta$-lactamase activity. All three mutants were plated alone or together with two densities of Ancestor cells under two CTX concentrations on M9 agar plates. The two tested CTX concentrations differed per mutant and were chosen to show single-cell establishment probabilities of about 50 and $80 \%$ compared to no CTX (Saebelfeld and Das et al., unpublished). Overnight cultures of the mutants were serially diluted to approximately $3 \times 10^{3}$ cells $/ \mathrm{ml}$, and the Ancestor overnight culture was serially diluted to $2 \times 10^{7}$ cells $/ \mathrm{ml}$ and $2 \times 10^{4}$ cells $/ \mathrm{ml}$. The mutant dilutions were then mixed 1:1 with either PBS or each of the two Ancestor density dilutions. Of those mixtures, $100 \mu \mathrm{l}$ aliquots were spread on M9 plates containing the respective CTX concentrations (15 replicates per condition), using a bacterial spreader, resulting in $\sim 150$ cells of the respective mutant strain plated with either $0, \sim 10^{3}$, or $\sim 10^{6}$ Ancestor cells. All used CTX concentrations were above the Ancestor MIC, allowing only for the mutant cells to grow into colonies. The plates were incubated at $37^{\circ} \mathrm{C}$ until the mutant

TABLE 1 | Overview of the used strains.

\begin{tabular}{|c|c|c|c|}
\hline $\begin{array}{l}\text { Wildtype strain } \\
\text { (without TEM } \\
\text { allele*) }^{\star}\end{array}$ & $\begin{array}{l}\text { TEM amino acid } \\
\text { substitutions }\end{array}$ & $\begin{array}{l}\text { MIC for } \\
\text { CTX } \\
(\mu \mathrm{g} / \mathrm{ml})\end{array}$ & $\begin{array}{l}\text { Strain name } \\
\text { (as used here) }\end{array}$ \\
\hline \multirow{4}{*}{$\begin{array}{l}\text { MG1655 DA28100 } \\
\text { galK::YFP } \triangle \text { CAT- or } \\
\text { galK::BFP } \triangle \text { CAT- }\end{array}$} & None (TEM-1) & 0.08 & Ancestor \\
\hline & G238S & 0.64 & Single mutant \\
\hline & E104K/G238S & 10.24 & Double mutant \\
\hline & E104K/M182T/G238S & 81.92 & Triple mutant \\
\hline
\end{tabular}

Each one of four TEM alleles with different cefotaxime (CTX) hydrolysing activity was inserted into an Escherichia coli wildtype strain.

MIC, minimum inhibitory concentration.

${ }^{*} \mathrm{MIC}$ of the Wildtype strain for CTX $=0.04 \mu \mathrm{g} / \mathrm{ml}$. colonies were big enough to count them unambiguously (20$40 \mathrm{~h}$ ); the number of colony-forming units (CFUs) per plate was counted under white light, using a digital colony counter. To ensure that all counted colonies were indeed mutants, the BFP-expressing Ancestor strain, and the YFP-expressing mutant strains were used for this experiment. Within the lowest CTX concentration treatment for the Single mutant, one replicate of each tested Ancestor density was excluded from the dataset, as overlapping colonies prevented unambiguous CFU counts. After colony counting, all plates were checked under a fluorescence microscope (LEICA M165 FC), using GFP and CFP filters for detecting the YFP and BFP signals, respectively. For the Single mutant, a single colony containing the BFP Ancestor fluorophore was found within the $10^{6}$ ancestral cells treatment in each one replicate of the two tested CTX concentrations. These two colonies were subtracted from the CFU counts.

\section{Interaction Experiment 2}

To test whether nutrient release from lysed cells and/or enzymatic breakdown of CTX by the Ancestor could explain the observed positive effect on mutant establishment probability in the first Interaction experiment, we conducted a second plating experiment. To test for a potential involvement of enzymatic breakdown, approximately 150 Triple mutant cells were introduced into populations of $\sim 10^{6}$ cells of either the Ancestor or the Single mutant (with $\sim 130$-fold higher catalytic efficiency against CTX relative to the Ancestor with the TEM1 allele, see Salverda et al., 2011) under the same two CTX concentrations as in the Interaction experiment 1 . In addition, the Triple mutant was introduced into populations of $\sim 10^{6}$ cells of heat-killed Ancestor or heat-killed Single mutant cells, to test whether nutrient addition from cell lysis alone could explain the observed pattern.

To set up the experiment, half of the overnight culture of each of the YFP-labeled Ancestor and Single mutant was heat-killed via incubation at $80^{\circ} \mathrm{C}$ for $3 \mathrm{~h}$ and then put on ice for $20 \mathrm{~min}$. An overnight culture of the BFP-labeled Triple mutant was serially diluted to $3 \times 10^{3}$ cells $/ \mathrm{ml}$, and the alive and heat-killed overnight cultures of the Ancestor and Single mutant were diluted to $2 \times 10^{7}$ cells $/ \mathrm{ml}$. The Triple mutant dilution was then mixed 1:1 with either PBS or the dilutions of alive or heat-killed Ancestor or alive Single mutant strains. $100 \mu \mathrm{l}$ aliquots of these mixtures were then spread on M9 plates containing 1.44 or $1.76 \mu \mathrm{g} / \mathrm{ml}$ CTX (i.e., the same concentrations as used for the Triple mutant in the first interaction experiment; equivalent to 0.018 and $0.021 \%$ of the Triple mutant's MIC), using $3 \mathrm{~mm}$ glass beads. As for the previous experiment, the used CTX concentrations were higher than the Ancestor and Single mutant MICs (see Table 1), so that only Triple mutant cells were expected to form colonies. The plates were incubated at $37^{\circ} \mathrm{C}$ until the colonies were big enough to count them unambiguously (16-20 h); the number of CFUs per plate was counted under white light, using a digital colony counter. To ensure that the counted colonies were Triple mutants, a fluorescence microscope was used as described above. No colonies expressing the YFP fluorophore (i.e., used for the Ancestor and Single mutant strains) were found. For each tested condition, 15 replicates were set up. To estimate the added 
number of cells per strain, the dilution containing only the Triple mutant was spread on 15 M9 plates without CTX. The alive Ancestor and alive Single mutant cell dilutions were further diluted to $10^{3}$ cells $/ \mathrm{ml} ; 200,100$ and $50 \mu \mathrm{l}$ aliquots of these dilutions were spread on three LB agar plates each. All plates were incubated overnight at $37^{\circ} \mathrm{C}$. Colony counts of the alive Ancestor and Single mutant cells showed that the initially added numbers in the experimental setup were comparable with $1.05 \times 10^{6}$ and $1.01 \times 10^{6}$ cells for each strain, respectively.

\section{Bioassay}

The antibiotic removal capacity of three strains was tested in a bioassay: the YFP-labeled Ancestor and Single mutant, as used in the Interaction experiment 2, and in addition the YFP-labeled Wildtype strain (Table 1). This Wildtype strain does not contain a TEM allele and thus does not display any $\beta$-lactamase activity, but is otherwise genetically identical to both the Ancestor and Single mutant strains. This means that it contains the same number of penicillin-binding proteins (PBPs), which is the main target for CTX and thus controls for the difference of CTX removal via breakdown and target binding.

Overnight cultures of each strain were diluted 1:10 into $1 \mathrm{ml}$ M9 medium (resulting in a cell density of $2 \times 10^{8}$ cells $/ \mathrm{ml}$ at the beginning of the assay), containing $50 \mu \mathrm{M}$ IPTG and $1.76 \mu \mathrm{g} / \mathrm{ml}$ CTX (i.e., the highest tested concentration from the agar experiments; note that due to the dilution via culture addition, the final tested CTX concentration in this assay is $1.58 \mu \mathrm{g} / \mathrm{ml}$ ) in three replicate tubes. In addition, heat-killed cells (as described above) of each strain were inoculated the same way. To control for spontaneous CTX breakdown under the tested conditions, three tubes were incubated in the absence of bacterial cells (CTX-only medium controls). For this, the M9 medium with CTX was mock-inoculated 1:10 with the M9 medium that was used for the overnight cultures the day before. All tubes were incubated for $3 \mathrm{~h}$ at $37^{\circ} \mathrm{C}$ and $250 \mathrm{rpm}$. Although the used CTX concentration was at least three-fold higher than the MIC of the strains (as determined previously in a standard MIC assay with $5 \times 10^{5}$ cells $/ \mathrm{ml}$, cf. section "Strains and Culture Conditions" and Table 1), all alive cultures showed growth after the 3-hour incubation. This is likely to be attributed to the socalled inoculum effect (Queenan et al., 2004), i.e., the increase of MIC with increasing inoculum size due to collective effects. The increase in cell density during the 3-hour incubation is important to take into account when determining the CTX removal capacity of the strains. We therefore estimate the final density of the cultures to be $\sim 10^{9}$ cells $/ \mathrm{ml}$ which is used for estimating the CTX removal capacity in the Results section "Modeling the Dynamic Interactions Between Highly Resistant and More Susceptible Strains".

After incubation, all tubes were centrifuged for $1 \mathrm{~min}$ at $4000 \mathrm{rpm}$. The supernatant was sterilized through a $0.2 \mu \mathrm{m}$ filter and used for setting up MIC assays. For this, the supernatant of each test tube was diluted in 1.35-fold steps with fresh M9 medium, containing $50 \mu \mathrm{M}$ IPTG. In total, 11 putative CTX concentrations (i.e., assuming no removal) ranging from 0.04 to $0.79 \mu \mathrm{g} / \mathrm{ml}$ were tested per culture in 96-well plates. All wells were inoculated with $\sim 10^{5}$ cells of the YFP-labeled Ancestor strain in a total volume of $200 \mu \mathrm{l}$ per well. Growth of this strain in higher putative CTX concentrations would indicate the removal of CTX from the medium during the initial 3-hour culture incubation. Controls included (i) a filtration control: per culture, undiluted supernatant was mock-inoculated with M9 $9_{\text {IPTG50 }}$ medium, i.e., without bacterial cells to control for removal of alive cells after filtration; (ii) a growth control: M9 $9_{\text {IPTG50 }}$ medium without CTX was inoculated with $\sim 10^{5}$ Ancestor cells; and (iii) a blank medium control consisting of M9 $_{\text {IPTG50 medium only. The 96- }}$ well plates were incubated for $18 \mathrm{~h}$ at $37^{\circ} \mathrm{C}$ (static). After incubation, $\mathrm{OD}$ at $600 \mathrm{~nm}$ of all wells was measured in a plate reader (Victor $3^{\mathrm{TM}}$, PerkinElmer) without the plate lid. None of the supernatant or blank medium controls showed growth. The threshold to determine growth was set to an OD of 0.1 .

Minimum inhibitory concentrations were determined as the highest putative CTX concentration that did not show growth. CTX removal was computed by finding the difference between the putative and real (based on the CTX-only medium controls) MIC and then adjusted by the relevant dilution factor, and finally by calculating the mean over the replicates. Standard errors (dilution-factor adjusted) of putative and real MICs were calculated based on the replicates; the error in concentration reduction was estimated as the square root of the sum of the squared errors of these two quantities.

\section{Statistical Analyses of the Interaction Experiments}

To test for a significant effect of the respective treatment on the number of mutant CFUs, two-way ANOVAs were performed. For the analysis of Interaction experiment 1, the CFU counts were square-root transformed and the effects of CTX concentration and added number of Ancestor cells were included as fixed factors, followed by a Tukey's HSD test. For the analysis of Interaction experiment 2, the effects of CTX concentration and background population were included as fixed factors after checking for homogeneous variances of the residuals and normal distribution of the data, followed by a Tukey's HSD test. All statistical analyses were conducted in R v.3.6.2 (R Core Team, 2019) with the car package (Fox and Weisberg, 2019) to perform Levene tests; Figures 1, 2 were produced using the package ggplot2 (Wickham, 2016).

\section{RESULTS}

\section{Susceptible Cells Facilitate the Establishment of $\beta$-Lactamase Mutants}

In a first plating experiment, we tested whether the presence of Ancestor cells affected the establishment of three TEM mutants with increasing CTX resistance at two CTX concentrations. Note that the Ancestor contains a $\beta$-lactamase with low activity toward CTX, so despite being commonly regarded as "susceptible" due to its small MIC (cf. Table 1), it still bears the potential to induce positive social interactions via antibiotic breakdown. Generally, the establishment (drift survival of single cells and outgrowth into visible colonies) of all three tested mutants 


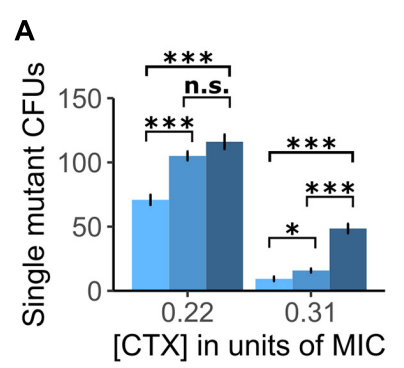

Added Ancestor cells
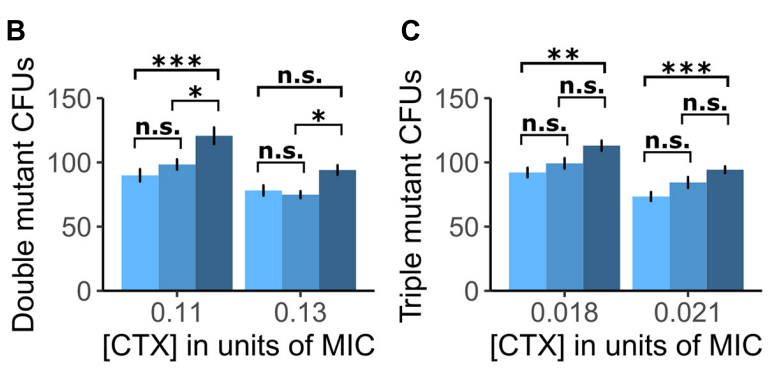

[CTX] in units of MIC

FIGURE 1 | The establishment of single cells into visible colonies of the three tested TEM mutants is shown as counts of colony-forming units (CFUs) of (A) the Single mutant, (B) the Double mutant, and (C) the Triple mutant under exposure to two cefotaxime (CTX) concentrations [shown as a fraction of each mutant strain's minimum inhibitory concentration (MIC)] in either the absence of Ancestor cells or the presence of Ancestor cells at two different densities. Significance levels based on Tukey's HSD tests are shown within CTX concentrations, between the number of added Ancestor cells after running two-way ANOVA's over the whole dataset per mutant strain (cf. Supplementary Table 1). n.s., not significant, ${ }^{*} p<0.05,{ }^{* *} p<0.01,{ }^{* * *} p<0.001$.

was increased when introduced into the Ancestor population (Figure 1), although this difference was not significant for all treatment comparisons (Supplementary Table 1). Moreover, establishment probability increased with Ancestral cell density under all conditions (Figure 1).

\section{Mutant Establishment Is Particularly Affected by Live Cells Expressing Active $\beta$-Lactamase}

In a subsequent plating experiment, we tested whether nutrient access via lysed cells or CTX removal via enzymatic breakdown explains the observed positive effect of relatively susceptible bacteria on the establishment of the resistant mutants. To do so, the Triple mutant was introduced into populations of either heat-killed or alive Ancestor or Single mutant cells under two CTX concentrations. When plated alone, the fraction of established Triple mutant cells, determined as CFUs, decreased by approximately $34 \%$ and $50 \%$ in the low and high CTX concentrations compared to no CTX, respectively (Figure 2). The addition of heat-killed Ancestor or Single mutant cells did not affect the establishment of the Triple mutant at either CTX concentration (Figure 2 and Supplementary Table 2). However, the addition of alive Ancestor cells with low $\beta$-lactamase activity significantly increased the establishment of the Triple mutant under both CTX concentrations. This increase was further pronounced by the presence of alive Single mutant cells (Figure 2 and Supplementary Table 2), suggesting an important role for enzymatic breakdown.

\section{Test of CTX Removal via Target Binding or Breakdown}

To confirm whether the increased establishment probability of the Triple mutant in the presence of alive cells (Figure 2) is due to CTX removal, and examine the contribution of CTX binding and hydrolysis during this process, we performed a bioassay to estimate CTX removal for the Wildtype (without TEM, i.e., no CTX-hydrolyzing activity), the Ancestor (with low hydrolyzing activity) and the Single mutant (with moderate

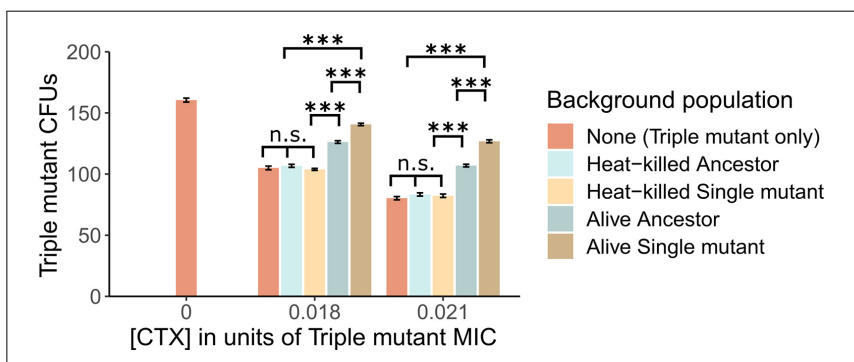

FIGURE 2 | The establishment of Triple mutant cells is shown as counts of CFUs under the exposure of two CTX concentrations (shown as a fraction of the Triple mutant's MIC), in the absence and presence of a "Background population": $10^{6}$ alive or heat-killed Ancestor cells, and alive or heat-killed Single mutant cells. In addition, the number of Triple mutant CFUs is shown in the absence of CTX. Significance levels based on Tukey's HSD tests are shown within CTX concentrations, between the type of Background population after running two-way ANOVA's over the whole dataset (cf. Supplementary Table 2). n.s., not significant, ${ }^{* * *} p<0.001$.

hydrolyzing activity) strains. We estimated the CTX removal rate of the three strains by culturing alive and heat-killed cells of each strain in medium with a supra-MIC CTX concentration for $3 \mathrm{~h}$ and then using the cell-free medium in a standard MIC assay with the Ancestral strain (see section "Materials and Methods"). Results show that the alive Ancestor and Single mutant removed $18.3( \pm 9.5) \%$ and $27.1( \pm 8.8) \%$ CTX from the liquid medium, respectively (Table 2 ). Neither the Wildtype strain (without $\beta$-lactamase activity) nor the heat-killed mutants (with inactivated $\beta$-lactamase activity) showed a decrease in CTX concentration (Table 2), supporting a significant role only for the $\beta$-lactamase in CTX removal.

\section{Modeling the Dynamic Interactions Between Highly Resistant and More Susceptible Strains}

Motivated by the observation that only strains expressing TEM $\beta$-lactamase reduce the concentration of CTX, we attempted to predict the amount of CTX removal from the agar medium 
in the experiment described above (Figure 2). In related work (Saebelfeld and Das et al., unpublished), we obtained an estimate of the establishment probability of the Triple mutant grown alone on agar under similar conditions as a continuous function of CTX concentration through curvefitting on a larger data set (Supplementary Figure 1). If we assume that the increased establishment probability in the presence of other cells in the experiment presented in Figure $\mathbf{2}$ is solely due to CTX breakdown, we can use the establishment probabilities to estimate the CTX reduction by finding the point on the curve corresponding to the increased establishment probability and its corresponding concentration (cf. Supplementary Figure 1). Using this, we estimate that the Ancestor reduces the concentration by $2.2 \%( \pm 2.6 \%)$ at $1.44 \mu \mathrm{g} / \mathrm{ml} \mathrm{CTX}$, and $16 \%( \pm 1.9 \%)$ at $1.76 \mu \mathrm{g} / \mathrm{ml} \mathrm{CTX}$, whereas the Single mutant reduces the concentration by $6.9 \%( \pm 2.9 \%)$ at $1.44 \mu \mathrm{g} / \mathrm{ml} \mathrm{CTX}$, and $20 \%( \pm 2.2 \%)$ at $1.76 \mu \mathrm{g} / \mathrm{ml}$ CTX. To compare with the bioassay, we do a simple linear interpolation to estimate that the Ancestor reduces the concentration by $8.2 \%( \pm 1.7 \%)$ and the Single mutant by $13 \%( \pm 1.9 \%)$ at $1.58 \mu \mathrm{g} / \mathrm{ml}$. These values are lower than the ones determined in the bioassay (Table 2); the reason for this may be that the estimate here assumes that the establishment process begins at a constant, reduced concentration, whereas in fact the concentration reduction likely occurs gradually by the activity of the background cells during establishment. This can lead to an underestimate of the concentration reduction here. However, our estimates also indicate that the CTX reduction by the Ancestral strain is surprisingly high, considering its approximate 130 -fold lower catalytic efficiency relative to the Single mutant.

To explain the unexpectedly high antibiotic removal by the Ancestor, we used a modeling approach. We begin with considering the difference in catalytic efficiency $\left(k_{\text {cat }} / K_{M}\right)$ of the Ancestor and Single mutant TEM variants. Based on reported estimates for both variants (Salverda et al., 2011) and assuming constant conditions, we predict a net reduction of CTX from the medium of $0.06 \%$ for the Ancestor and $7.5 \%$ for the Single

TABLE 2 | Cefotaxime removal from liquid medium in the bioassay (from the initial $1.58 \mu \mathrm{g} / \mathrm{ml}$ ) by strains expressing different $\beta$-lactamase alleles or no $\beta$-lactamase, after $3 \mathrm{~h}$ of incubation.

\begin{tabular}{|c|c|c|c|}
\hline Strain & Culture condition & $\begin{array}{l}\text { CTX removal } \\
{[\mu \mathrm{g} / \mathrm{ml}]}\end{array}$ & CTX removal [\%] \\
\hline \multirow{2}{*}{$\begin{array}{l}\text { Ancestor (low } \\
\beta \text {-lactamase } \\
\text { activity) }\end{array}$} & Alive cells & $0.29( \pm 0.15)$ & $18.3( \pm 9.5)$ \\
\hline & Heat-killed cells & 0 & 0 \\
\hline \multirow{2}{*}{$\begin{array}{l}\text { Single mutant } \\
\text { (moderate } \\
\beta \text {-lactamase } \\
\text { activity) }\end{array}$} & Alive cells & $0.43( \pm 0.14)$ & $27.1( \pm 8.8)$ \\
\hline & Heat-killed cells & 0 & 0 \\
\hline \multirow{2}{*}{$\begin{array}{l}\text { Wildtype (no } \\
\beta \text {-lactamase } \\
\text { activity) }\end{array}$} & Alive cells & 0 & 0 \\
\hline & Heat-killed cells & 0 & 0 \\
\hline
\end{tabular}

mutant (Supplementary Material 3). While the prediction for the Single mutant can be considered roughly consistent with our experimental finding (given the crude nature of the estimate and the data), the prediction for the Ancestor is two orders of magnitude smaller and inconsistent with its observed only onethird smaller reduction (Table 2). Indeed, given that the ancestral TEM-1 has very low activity, it is surprising that it achieves even a measurable reduction. One possible explanation is that the ancestral enzyme (TEM-1), while breaking down the antibiotics at a very low rate, nonetheless binds the antibiotic at a high rate (Queenan et al., 2004). However, this explanation seems unlikely, because removal by binding happens only for one antibiotic molecule per enzyme molecule in the periplasmic space, whereas the turnover rate due to degradation by the Single mutant is about 810 antibiotic molecules per enzyme molecule in $30 \mathrm{~min}$ (which is the scale of cell division time; the number of antibiotic molecules degraded is obtained from the reaction rate estimates in Supplementary Material 3). Thus, binding without hydrolysis cannot explain the finding that the Ancestor and Single mutant remove CTX at comparable rates.

To investigate this issue further, we used a model introduced in Geyrhofer and Brenner (2020) which incorporates not just enzyme kinetics but also cell growth and death, and the exchange of enzyme and antibiotics between cells and the environment. Details on the dynamical model can be found in Supplementary Material 4. In short, this model presents a more realistic picture of the dynamical process underlying antibiotic removal. Cell numbers decrease initially at high antibiotic concentration, while simultaneously removing antibiotic through $\beta$-lactamase synthesis and secretion. Depending on the inoculum size, the decrease in antibiotic concentration in the medium can lead to a resurgence in cell growth at a later time (see Figure 3). The model has a multi-dimensional parameter space that can be explored to locate the source of the unexpectedly high antibiotic removal by the Ancestral strain. For the numerical simulation (Supplementary Material 3) we chose $1.6 \mu \mathrm{g} / \mathrm{ml}$ CTX, which is the mean of the two highest concentrations in the plating experiments and is approximately equal to the bioassay concentration $(1.584 \mu \mathrm{g} / \mathrm{ml})$. Many of the parameters in the model can be either determined from the literature or have an insignificant impact on the results (Supplementary Material 4). However, two important parameters that are left undetermined are $\sigma_{B}$ and $\sigma_{E}$, which are the rate constants for the flux of antibiotic and enzyme, respectively, between the cells and the environment.

One possibility is that the enzyme synthesis and/or permeability parameters $\sigma_{B}$ and $\sigma_{E}$ differ for the Ancestor and Single mutant. For example, $\sigma_{B}$ and $\sigma_{E}$ could be higher in the Ancestor due to higher rates of cell wall defects (induced by the typical interference of $\beta$-lactam antibiotics with cell wall synthesis, see e.g., Yao et al., 2012), although there is no empirical support for this to our knowledge. However, numerical simulations show that the discrepancy is not explained even when $\sigma_{E}$ and $\sigma_{B}$ are two orders of magnitude higher for the Ancestor (see Supplementary Figure $\mathbf{2}$ for exploration of the parameter space). As this phenomenon by itself does not provide a satisfactory explanation, we considered a possible 


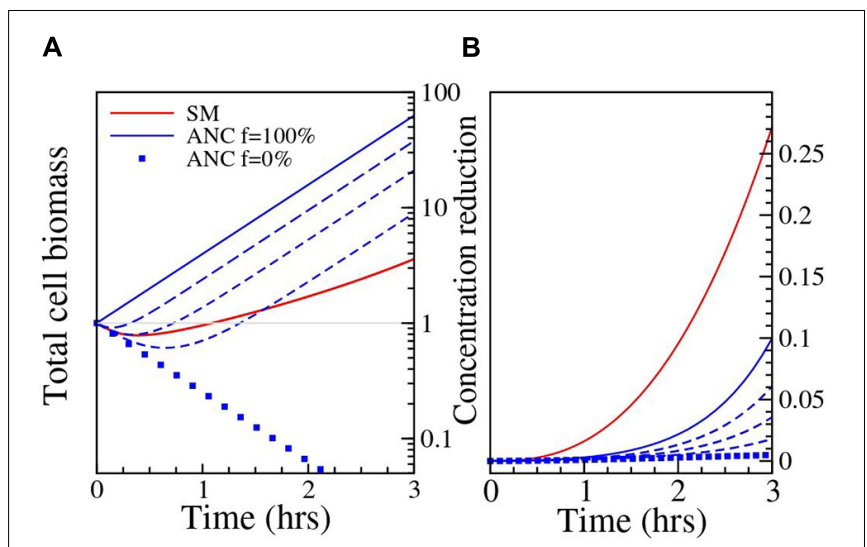

FIGURE 3 | Results of the numerical simulation. Parameters: Ancestor (ANC) $\sigma_{E}=16 h^{-1} ; \sigma_{B}=9.2 h^{-1}$; Single mutant (SM) $\sigma_{E}=2.81 h^{-1}$; $\sigma_{B}=1 h^{-1}$. Filamentation has been quantified through a normalized filamentation rate $f$ (see Supplementary Material 4); the used values of $f$ are 0\% (filled squares), 25\%, 50\%, 75\% (dashed lines) and 100\% (solid line).

(A) The change in biomass (in units of single cell size) is shown as a function of time. The blue lines correspond to the Ancestor and show the increasing effect of filamentation on cell biomass growth. For the Single mutant and the Ancestor at $f=25 \%$, the cells die initially and then grow again as the antibiotic concentration is reduced through breakdown. (B) The reduction in antibiotic concentration relative to the initial value is shown as a function of time.

additional role of cell filamentation. We know that our E. coli strain undergoes filamentation under CTX exposure, an effect induced via SOS response (Justice et al., 2008). Both the rate of filamentation, i.e., the fraction of the population that undergoes filamentation (Jeffrey Power, personal communication), and time of subsequent cell lysis (Zahir et al., 2020) are strongly straindependent. Further, during filamentation, the biomass of the cells keeps growing, even though cell division does not occur due to inhibited septum formation (Pogliano et al., 1997). Filamenting cells, therefore, continue to synthesize enzyme that breaks down the antibiotic, even if many of them eventually lyse and die (Zahir et al., 2020). If we assume that in our experiments the Ancestor cells do filament (i.e., show delayed lysis, which in turn keeps the cells available to produce enzyme over a longer duration) and that enzyme production is proportional to biomass growth, whereas this is not the case for the Single mutant (i.e., it has a lower filamentation rate and thus earlier cell lysis which leads to a relatively lower enzyme production compared to the Ancestor), this can be incorporated in the model by setting the growth rate parameter of the Ancestor to be independent of the antibiotic concentration. Under this assumption, the breakdown by the Ancestor becomes comparable to that of the Single mutant in the relevant parameter range. For $\sigma_{E}=2.81 h^{-1}, \sigma_{B}=1 h^{-1}$, the reduction is $1.8 \%$, which is significantly higher than the value obtained without filamentation, but still lower than what is expected from the bioassay data (see Supplementary Figure 2). But moderately higher values of enzyme expression and release produce better results. For example, with $\sigma_{E}=16 h^{-1}$, $\sigma_{B}=9.2 h^{-1}$ we get a $10 \%$ reduction. The simulated reduction of antibiotic concentration by filamenting ancestral cells is shown in Figure 3.

\section{DISCUSSION}

A crucial moment during the emergence of antibiotic resistance is the establishment, i.e., the survival of stochastic drift loss and outgrowth into a population, of an initial single mutant cell. Here, we examined whether and how the establishment of de novo antibiotic-resistant mutant cells was affected by social interactions with populations of more susceptible cells. The successful establishment of a mutant cell was determined by its outgrowth into a colony-forming unit, visible to the naked eye. In the first plating experiment, we showed that the establishment probability of three separately tested TEM $\beta$-lactamase mutants with increasing hydrolysis activity against the cephalosporin CTX was increased when introduced into populations of a strain expressing the ancestral TEM-1 allele with very low $\beta$-lactamase activity, under exposure to two CTX concentrations. We next hypothesized that three, mutually not exclusive, mechanisms could be involved in this effect: (i) additional nutrients were provided by dying Ancestor cells, (ii) Ancestor cells depleted antibiotic molecules in the environment via binding to their cellular target sites and (iii) Ancestor cells reduced the environmental antibiotic concentration via $\beta$-lactamase-induced CTX hydrolysis. In the following, we discuss all mechanisms and their potential role in our system.

It has been shown previously that the amount of nutrients can play a role during establishment. When antibiotic-resistant E. coli mutants were plated on agar, containing different antibiotics, Coates et al. (2018) found that their establishment was lower in a poor than in a rich nutrition environment due to a reduction in growth rate. Further, as shown repeatedly, contents released from decaying bacterial cells can be reutilised by growing cells (e.g., Koch, 1959; Banks and Bryers, 1990; Rozen et al., 2009). As in our setup, the Ancestor cells died in the presence of the antibiotic via cell lysis (Cho et al., 2014) and we used minimal medium, it is conceivable that extra nutrients released from dying Ancestor cells supported mutant establishment. To test this idea, we set up a subsequent plating experiment where we used populations of heat-killed Ancestor and Single mutant cells, in which Triple mutant cells were introduced for testing their establishment. Heat-killing was chosen to inactivate the $\beta$-lactamases produced by both strains as hydrolyzing enzymes can further break down antibiotics after cell death (Udekwu et al., 2009; Lenhard and Bulman, 2019). To sustain one cell, an estimated 110 heat-killed E. coli cells are needed to provide sufficient nutrients (Nioh and Furusaka, 1968). Thus, some 16,500 heat-killed Ancestor or Single mutant cells would have been needed to sustain the growth of the 150 plated Triple mutant cells under starving conditions. The absence of an effect of $10^{6}$ added heat-killed cells on the establishment of the Triple mutant (Figure 2) suggests that the addition of nutrients due to cell lysis plays a negligible role under the tested conditions, leaving CTX removal from the environment as the most probable explanation for the observed positive effect on mutant establishment. However, we cannot rule 
out that our method of heat-killing causes only a fraction of the cells to lyse and release nutrients (Smakman and Hall, 2020).

A positive effect of susceptible cells on the establishment of de novo antibiotic-resistance conferring mutants has been reported before for $P$. aeruginosa in the presence of streptomycin and meropenem (Alexander and MacLean, 2020). The authors speculated that the mechanism for this effect was the binding of the antibiotics to components of the susceptible cells, thereby reducing the antibiotic concentration in the environment (Udekwu et al., 2009; Abel Zur Wiesch et al., 2015). The main target for streptomycin is ribosomes (Demirci et al., 2013), a major cell component with numbers ranging between a few thousand to several ten-thousands per cell, depending on growth rate and species (Martin and Iandolo, 1975; Bremer and Dennis, 1996; Barrera and Pan, 2004; Leskelä et al., 2005). CTX on the other hand predominantly binds to the penicillin-binding protein PBP3 (Jones and Thornsberry, 1982; Piras et al., 1990; Cho et al., 2014), which is present at only about 60 to 130 units per E. coli cell, depending on the medium and growth rate (Dougherty et al., 1996). Thus, antibiotic reduction via binding to its cellular target can be expected to be much lower in our experimental system compared to that of Alexander and MacLean (2020). Indeed, we found that the Wildtype strain without $\beta$-lactamase, but with the same number of PBPs, was not able to remove detectable amounts of CTX from the liquid medium in the bioassay (Table 2). This indicates that the removal of CTX via binding to cellular components is negligible in our system, leaving CTX removal by enzymatic breakdown the most likely mechanism for mutant establishment support.

Though the $\beta$-lactamase activity of the Ancestor against CTX is very low, it may have reduced the environmental CTX concentration in the first plating experiment sufficiently by catalyzing its hydrolysis, thereby supporting the establishment of the three resistant mutants. To test whether CTX breakdown via $\beta$-lactamase activity played a role in this effect, we introduced the Triple mutant into populations of the moderately active Single mutant compared to the lowly active Ancestor. Indeed, the establishment of the Triple mutant was further increased in the presence of the Single mutant compared to the Ancestor (see Figure 2), indicating that hydrolysis of the antibiotic contributes to the observed effect. This was further supported by the results of the bioassay, where both the Ancestor and the Single mutant were able to remove CTX from the liquid medium, whereas the Single mutant removed more of the antibiotic than the Ancestor (Table 2). However, the CTX removal rate by the Ancestor and its effect on Triple mutant establishment are unexpectedly high when compared with the Single mutant. Specifically, the Ancestor removed about two-thirds of the amount of CTX (Table 2) and restored roughly half of the lost established Triple mutant cells compared to the Single mutant (Figure 2), despite its 130-fold lower catalytic efficiency against CTX (Salverda et al., 2011). Thus, CTX hydrolysis based on enzyme kinetics alone cannot explain the observed effect of the Ancestor. To investigate this further, we adapted a model introduced by Geyrhofer and Brenner (2020), where the effect of cell filamentation was incorporated by allowing the biomass of cells to grow without cell division in the presence of the antibiotic. Exploration of various scenarios using this model indicates that delayed lysis of filamentous Ancestor cells, coupled with an increased $\beta$-lactamase release due to cell wall permeability which leads to higher $\beta$-lactamase synthesis, may explain the unexpected high breakdown of CTX by the Ancestor. While these results are not conclusive, they do point to interesting directions for future work.

Understanding the factors that determine the probability of antibiotic-resistant bacterial cells establishing into populations is important in the clinical context. The maintenance of resistant cells within sensitive populations in the absence of antibiotics leads to a higher risk of colonization by the mutant when antibiotics are applied and resources are freed up by the dying, more susceptible, cells (De Roode et al., 2004; Pollitt et al., 2014; Olsen, 2015). Our results indicate that this emergence could be further supported when the antibiotic is broken down. Apart from hydrolyzing enzymes like $\beta$-lactamases, those systems could include other antibiotics that are modified or degraded, such as erythromycin, tetracyclines and chloramphenicol (Nicoloff and Andersson, 2016).

In conclusion, we showed that the removal of CTX from the environment by bacterial cells expressing $\beta$-lactamase enzymes with relatively low activity against the antibiotic enhanced the establishment probability of mutants with more active enzymes. We believe that similar positive effects may affect the establishment of mutants resistant to other antibiotics, as was shown for streptomycin (Alexander and MacLean, 2020), but the size of this effect will depend on systemspecific factors, including the antibiotic target, the resistance mechanism, and the ability of the strain for filamentation. Notably, the positive effect of relatively susceptible cells on the initial establishment of resistant mutants we report contrasts with their later impeding influence by preventing the fixation of higher resistance mutants once sufficient antibiotic has been removed (Yurtsev et al., 2013). Our study is among few demonstrating the importance of the resistance mechanism and conditions during the intial steps of antibiotic resistance emergence. Further investigations on factors contributing to the establishment of de novo resistance mutants in other systems would contribute to a better understanding of the longterm persistence and re-emergence of infections with resistant pathogens in clinical settings.

\section{DATA AVAILABILITY STATEMENT}

The original contributions presented in the study are included in the article/Supplementary Material, further inquiries can be directed to the corresponding author.

\section{AUTHOR CONTRIBUTIONS}

MS, JB, and AH conducted the experiments. SD developed the model with help from JK. MS and SD wrote the manuscript with help from JK and JV. All authors conceived the study, read the manuscript, and approved the final version. 


\section{FUNDING}

This work was supported by the German Research Foundation (DFG; CRC 1310 Predictability in Evolution).

\section{ACKNOWLEDGMENTS}

We would like to thank Jeffrey Power for helpful discussions on the manuscript and insight into his data, and Helen Alexander as well as two reviewers for their helpful suggestions on improving the manuscript. A previous version of this work is available as a preprint on bioRxiv, doi: 10.1101/2021.04.20.440616.

\section{REFERENCES}

Abel Zur Wiesch, P., Abel, S., Gkotzis, S., Ocampo, P., Engelstädter, J., Hinkley, T., et al. (2015). Classic reaction kinetics can explain complex patterns of antibiotic action. Sci. Transl. Med. 7, 1-12. doi: 10.1126/scitranslmed.aaa8760

Alexander, H. K., and MacLean, R. C. (2020). Stochastic bacterial population dynamics restrict the establishment of antibiotic resistance from single cells. Proc. Natl. Acad. Sci. U.S.A. 117, 19455-19464. doi: 10.1073/pnas.1919672117

Aslam, B., Wang, W., Arshad, M. I., Khurshid, M., Muzammil, S., Rasool, M. H., et al. (2018). Antibiotic resistance: a rundown of a global crisis. Infect. Drug Resist. 11, 1645-1658. doi: 10.2147/IDR.S173867

Banks, M. K. and Bryers, J. D. (1990). Cryptic growth within a binary microbial culture. Appl. Microbiol. Biotechnol. 33, 596-601. doi: 10.1007/BF00172558

Barrera, A., and Pan, T. (2004). Interaction of the Bacillus subtilis RNase P with the 30S ribosomal subunit. RNA 10, 482-492. doi: 10.1261/rna.5163104

Baym, M., Stone, L. K., and Kishony, R. (2016). Multidrug evolutionary strategies to reverse antibiotic resistance. Science 351:aad3292. doi: 10.1126/science.aad3292

Blair, J. M. A., Webber, M. A., Baylay, A. J., Ogbolu, D. O., and Piddock, L. J. V. (2015). Molecular mechanisms of antibiotic resistance. Nat. Rev. Microbiol. 13, 42-51. doi: $10.1038 /$ nrmicro3380

Bremer, H., and Dennis, P. P. (1996). Modulation of chemical composition and other parameters of the cell by growth rate. EcoSal Plus 2, 1553-1569. doi: 10.1128/ecosal.5.2.3

Brown, S. P., West, S. A., Diggle, S. P., and Griffin, A. S. (2009). Social evolution in micro-organisms and a Trojan horse approach to medical intervention strategies. Philos. Trans. R. Soc. B Biol. Sci. 364, 3157-3168. doi: 10.1098/rstb. 2009.0055

Chelo, I. M., Nédli, J., Gordo, I., and Teotónio, H. (2013). An experimental test on the probability of extinction of new genetic variants. Nat. Commun. 4:2417. doi: $10.1038 /$ ncomms 3417

Cho, H., Uehara, T., and Bernhardt, T. G. (2014). Beta-lactam antibiotics induce a lethal malfunctioning of the bacterial cell wall synthesis machinery. Cell 159, 1300-1311. doi: 10.1016/j.cell.2014.11.017

Coates, J., Park, B. R., Le, D., Şimşek, E., Chaudhry, W., and Kim, M. (2018). Antibiotic-induced population fluctuations and stochastic clearance of bacteria. Elife 7:e32976. doi: 10.7554/eLife.32976

De Roode, J. C., Culleton, R., Bell, A. S., and Read, A. F. (2004). Competitive release of drug resistance following drug treatment of mixed Plasmodium chabaudi infections. Malar. J. 3:33. doi: 10.1186/1475-2875-3-33

Demirci, H., Murphy, F., Murphy, E., Gregory, S. T., Dahlberg, A. E., and Jogl, G. (2013). A structural basis for streptomycin-induced misreading of the genetic code. Nat. Commun. 4:1355. doi: 10.1038/ncomms2346

Dougherty, T. J., Kennedy, K., Kessler, R. E., and Pucci, M. J. (1996). Direct quantitation of the number of individual penicillin-binding proteins per cell in Escherichia coli. J. Bacteriol. 178, 6110-6115. doi: 10.1128/jb.178.21.6110-6115. 1996

Fox, J., and Weisberg, S. (2019). An $\{R\}$ Companion to Applied Regression, 3rd Edn. Thousand Oaks, CA: Sage.

Furusawa, C., Horinouchi, T., and Maeda, T. (2018). Toward prediction and control of antibiotic-resistance evolution. Curr. Opin. Biotechnol. 54, 45-49. doi: 10.1016/j.copbio.2018.01.026

\section{SUPPLEMENTARY MATERIAL}

The Supplementary Material for this article can be found online at: https://www.frontiersin.org/articles/10.3389/fmicb. 2021.698970/full\#supplementary-material

Supplementary Material 1 | ANOVAs and post hoc tests of the Interaction experiments.

Supplementary Material 2 | Determining establishment probability and the corresponding concentration.

Supplementary Material 3 | Expected CTX reduction in the bioassay.

Supplementary Material 4 | Dynamical model.

Gerrish, P. J., and Lenski, R. E. (1998). The fate of competing beneficial mutations in an asexual population. Genetica 102, 127-144. doi: 10.1007/978-94-0115210-5_12

Geyrhofer, L., and Brenner, N. (2020). Coexistence and cooperation in structured habitats. BMC Ecol. 20:14. doi: 10.1186/s12898-02000281-y

Gifford, D. R., De Visser, J. A. G. M., and Wahl, L. M. (2013). Model and test in a fungus of the probability that beneficial mutations survive drift. Biol. Lett. 9:20120310. doi: 10.1098/rsbl.2012.0310

Gullberg, E., Albrecht, L. M., Karlsson, C., Sandegren, L., and Andersson, D. I. (2014). Selection of a multidrug resistance plasmid by sublethal levels of antibiotics and heavy metals. MBio 5, 19-23. doi: 10.1128/mBio.01918-14. Editor

Haldane, J. B. S. (1927). A mathematical theory of natural and artificial selection, Part V: selection and mutation. Math. Proc. Cambridge Philos. Soc. 23, 838-844. doi: 10.1017/S0305004100015644

IACG (2019). No Time to Wait: Securing the Future From Drug-Resistant Infections. Report to the Secretary-General of the United Nations. doi: 10.1017/ s0305004100015644

Jones, R. N., and Thornsberry, C. (1982). Cefotaxime: a review of in vitro antimicrobial properties and spectrum of activity. Rev. Infect. Dis. 4(Suppl.), S300-S315. doi: 10.1093/clinids/4.supplement_2.s300

Justice, S. S., Hunstad, D. A., Cegelski, L., and Hultgren, S. J. (2008). Morphological plasticity as a bacterial survival strategy. Nat. Rev. Microbiol. 6, 162-168. doi: $10.1038 /$ nrmicro 1820

Koch, A. L. (1959). Death of bacteria in growing culture. J. Bacteriol. 77, 623-629. doi: $10.1128 /$ jb.77.5.623-629.1959

Lenhard, J. R., and Bulman, Z. P. (2019). Inoculum effect of $\beta$-lactam antibiotics. J. Antimicrob. Chemother. 74, 2825-2843. doi: 10.1093/jac/dkz226

Leskelä, T., Tilsala-Timisjärvi, A., Kusnetsov, J., Neubauer, P., and Breitenstein, A. (2005). Sensitive genus-specific detection of Legionella by a 16S rRNA based sandwich hybridization assay. J. Microbiol. Methods 62, 167-179. doi: 10.1016/ j.mimet.2005.02.008

Levy, S. F., Blundell, J. R., Venkataram, S., Petrov, D. A., Fisher, D. S., and Sherlock, G. (2015). Quantitative evolutionary dynamics using high-resolution lineage tracking. Nature 519, 181-186. doi: 10.1038/nature14279

Martin, S. E., and Iandolo, J. J. (1975). Translational control of protein synthesis in Staphylococcus aureus. J. Bacteriol. 122, 1136-1143. doi: 10.1128/jb.122.3.11361143.1975

Medaney, F., Dimitriu, T., Ellis, R. J., and Raymond, B. (2016). Live to cheat another day: bacterial dormancy facilitates the social exploitation of $\beta$-lactamases. ISME J. 10, 778-787. doi: 10.1038/ismej.2015.154

Nicoloff, H., and Andersson, D. I. (2016). Indirect resistance to several classes of antibiotics in cocultures with resistant bacteria expressing antibiotic-modifying or -degrading enzymes. J. Antimicrob. Chemother. 71, 100-110. doi: 10.1093/ $\mathrm{jac} / \mathrm{dkv} 312$

Nioh, I., and Furusaka, C. (1968). Growth of bacteria in the heat-killed cell suspensions of the same bacteria. J. Gen. Appl. Microbiol. 14, 373-385. doi: 10.2323/jgam.14.373

Olsen, I. (2015). Biofilm-specific antibiotic tolerance and resistance. Eur. J. Clin. Microbiol. Infect. Dis. 34, 877-886. doi: 10.1007/s10096-015-2323-z 
Palmer, A. C., and Kishony, R. (2013). Understanding, predicting and manipulating the genotypic evolution of antibiotic resistance. Nat. Rev. Genet. 14, 243-248. doi: $10.1038 / \operatorname{nrg} 3351$

Patwa, Z., and Wahl, L. M. (2008). The fixation probability of beneficial mutations. J. R. Soc. Interface 5, 1279-1289. doi: 10.1098/rsif.2008.0248

Piras, G., El Kharroubi, A., Van Beeumen, J., Coeme, E., Coyette, J., and Ghuysen, J. M. (1990). Characterization of an Enterococcus hirae penicillin-binding protein 3 with low penicillin affinity. J. Bacteriol. 172, 6856-6862. doi: 10.1128/ jb.172.12.6856-6862.1990

Pogliano, J., Pogliano, K., Weiss, D. S., Losick, R., and Beckwith, J. (1997). Inactivation of FtsI inhibits constriction of the FtsZ cytokinetic ring and delays the assembly of FtsZ rings at potential division sites. Proc. Natl. Acad. Sci. U.S.A. 94, 559-564. doi: 10.1073/pnas.94.2.559

Pollitt, L. C., Huijben, S., Sim, D. G., Salathé, R. M., Jones, M. J., and Read, A. F. (2014). Rapid response to selection, competitive release and increased transmission potential of artesunate-selected Plasmodium chabaudi malaria parasites. PLoS Pathog 10:e1004019. doi: 10.1371/journal.ppat.1004019

Queenan, A. M., Foleno, B., Gownley, C., Wira, E., and Bush, K. (2004). Effects of inoculum and beta-lactamase activity in AmpC- and extended-spectrum beta-lactamase (ESBL)-producing Escherichia coli and Klebsiella pneumoniae clinical isolates tested by using NCCLS ESBL methodology. J. Clin. Microbiol. 42, 269-275. doi: 10.1128/JCM.42.1.269

R Core Team (2019). R: A Language and Environment for Statistical Computing. Vienna: R Foundation for Statistical Computing.

Rozen, D. E., De Visser, J. A. G. M., and Gerrish, P. J. (2002). Fitness effects of fixed beneficial mutations in microbial populations. Curr. Biol. 12, 1040-1045. doi: 10.1016/S0960-9822(02)00896-5

Rozen, D. E., Philippe, N., De Visser, J. A., Lenski, R. E., and Schneider, D. (2009). Death and cannibalism in a seasonal environment facilitate bacterial coexistence. Ecol. Lett. 12, 34-44. doi: 10.1111/j.1461-0248.2008.01257.x

Salverda, M. L. M., Dellus, E., Gorter, F. A., Debets, A. J. M., van der Oost, J., Hoekstra, R. F., et al. (2011). Initial mutations direct alternative pathways of protein evolution. PLoS Genet. 7:e1001321. doi: 10.1371/journal.pgen.1001321

Schenk, M. F., Szendro, I. G., Krug, J., and de Visser, J. A. G. M. (2012). Quantifying the adaptive potential of an antibiotic resistance enzyme. PLoS Genet. 8:e1002783. doi: 10.1371/journal.pgen.1002783
Smakman, F., and Hall, A. R. (2020). Exposure to lysed bacteria can promote or inhibit growth of neighbouring live bacteria depending on local abiotic conditions. bioRxiv [Preprint]. doi: 10.1101/2020.10.23.352005

Udekwu, K. I., Parrish, N., Ankomah, P., Baquero, F., and Levin, B. R. (2009). Functional relationship between bacterial cell density and the efficacy of antibiotics. J. Antimicrob. Chemother. 63, 745-757. doi: 10.1093/jac/dkn554

Wickham, H. (2016). ggplot2: Elegant Graphics for Data Analysis. New York, NY: Springer-Verlag.

Yao, Z., Kahne, D., and Kishony, R. (2012). Distinct single-cell morphological dynamics under beta-lactam antibiotics. Mol. Cell 48, 705-712. doi: 10.1016/ j.molcel.2012.09.016

Yurtsev, E. A., Chao, H. X., Datta, M. S., Artemova, T., and Gore, J. (2013). Bacterial cheating drives the population dynamics of cooperative antibiotic resistance plasmids. Mol. Syst. Biol. 9:683. doi: 10.1038/msb.2013.39

Zahir, T., Wilmaerts, D., Franke, S., Weytjens, B., Camacho, R., Marchal, K., et al. (2020). Image-based dynamic phenotyping reveals genetic determinants of filamentation-mediated $\beta$-lactam tolerance. Front. Microbiol. 11:374. doi: $10.3389 /$ fmicb. 2020.00374

Conflict of Interest: The authors declare that the research was conducted in the absence of any commercial or financial relationships that could be construed as a potential conflict of interest.

Publisher's Note: All claims expressed in this article are solely those of the authors and do not necessarily represent those of their affiliated organizations, or those of the publisher, the editors and the reviewers. Any product that may be evaluated in this article, or claim that may be made by its manufacturer, is not guaranteed or endorsed by the publisher.

Copyright (c) 2021 Saebelfeld, Das, Brink, Hagenbeek, Krug and de Visser. This is an open-access article distributed under the terms of the Creative Commons Attribution License (CC BY). The use, distribution or reproduction in other forums is permitted, provided the original author(s) and the copyright owner(s) are credited and that the original publication in this journal is cited, in accordance with accepted academic practice. No use, distribution or reproduction is permitted which does not comply with these terms. 